\title{
Cross-contamination in Molecular Diagnostic Laboratories in Low- and Middle-income Countries: A Challenge to COVID-19 Testing
}

\author{
Pia Marie Albano, ${ }^{1}$ Kin Israel Notarte, ${ }^{2}$ Imee Macaranas, ${ }^{2}$ Benedict Maralit ${ }^{3}$ \\ ${ }^{1}$ Molecular Diagnostics Research Group, Research Center for the Natural and Applied Sciences, University of Santo Tomas, Manila, Philippines \\ ${ }^{2}$ Faculty of Medicine and Surgery, University of Santo Tomas, Manila, Philippines \\ ${ }^{3}$ DNA Sequencing Core Facility, Philippine Genome Center, University of the Philippines-Diliman, Quezon City, Philippines
}

\section{ABSTRACT}

At the start of the pandemic, the Philippines had to send swab samples to the Victorian Infectious Diseases Reference Laboratory in Melbourne, Australia for COVID-19 confirmation. With the increasing number of suspected cases needing confirmatory diagnostic testing, there was a demand to rapidly expand the capacity for widescale testing. Remarkably, within 200 days from announcement of the first confirmed COVID-19 case in the Philippines in January 30, 2020, the country has been able to expand its testing capacity from one national reference laboratory, the Research Institute for Tropical Medicine (RITM), to more than 100 licensed reverse transcription-polymerase chain reaction (RT-PCR) and cartridge-based PCR laboratories across the country. Due to the shortage of a trained clinical laboratory workforce, diagnostic centers are forced to hire additional personnel who have limited experience and technical knowledge and skills of molecular assays, especially in processing specimens, interpreting the results, identifying errors, and troubleshooting, in order to meet the demand of increased testing. Thus, the vulnerability to diagnostic errors, including cross-contamination, is increased and with the tendency for generating falsepositive results that can compromise the health of the patient and disrupt the efficacy of public health policies and public health response, surveillance programs, and restrictive measures for containing the outbreak. Hence, this review article aims to present the different sources of contamination in the laboratory setting where RT-PCR assays are conducted, as well as provide efficient, effective and feasible solutions to address these issues, most especially in low- and middle-income countries (LMICs) like the Philippines.

Key words: SARS-CoV-2; LMICs; RT-PCR, cross-contaminations, quality control, COVID-19

ISSN 2507-8364 (Online)

Printed in the Philippines.

Copyright $(2020$ by the PJP.

Received: 17 August 2020.

Accepted: 27 October 2020.

Published online first: 4 November 2020.

https://doi.org/10.21141/PJP.2020.09

Corresponding author: Pia Marie Albano, PhD

E-mail:psalbano@ust.edu.ph

\section{INTRODUCTION}

The emergence of the coronavirus disease 2019 (COVID-19) has caused a global public health emergency. The rapid escalation in the number of infections resulted in widescale shortages of personal protective equipment (PPE), diagnostic test kits, and essential equipment for patient treatment such as respirators. In resourcelimited countries like the Philippines and Indonesia, the overwhelming influx of severe COVID-19 cases has restricted testing to those who have severe symptoms and needing hospitalization. ${ }^{1,2}$ Consequently, the inability to rapidly expand the capacity for widescale testing has hindered response efforts. Moreover, the limited efforts for rapid contact tracing even in the absence of diagnostic testing could have also contributed to the rapid transmission of the virus.

Testing of samples for COVID-19 diagnosis is an integral part in resolving the current pandemic. The efforts of the Department of Health (DOH), Research Institute for Tropical Medicine (RITM), the University of the Philippines (UP), Philippine National Red Cross (PNRC) and the World Health Organization (WHO) with respect to building laboratory networks, providing training and proficiency testing, licensing labs, establishing networks for distributed testing, logistics, and addressing the challenges of identifying, validating, and approving test kits for use in the Philippines have been impressive. 
Prior to the pandemic, the expertise and capabilities to test for COVID-19 and similar entities were mostly coming from the academe and research centers. In addition, lockdown restrictions hindered molecular biologists from volunteering their services. When specimen collection was heightened, backlogs in testing were experienced as molecular diagnostic laboratories were being constructed across the country.

At the start of the pandemic, the Philippines had to send swab samples to the Victorian Infectious Diseases Reference Laboratory in Melbourne, Australia for COVID-19 confirmation. ${ }^{3}$ Remarkably, within two hundred days from announcement of the first confirmed COVID-19 case in the Philippines in January 30,2020 , the country has been able to expand its testing capacity from one national reference laboratory (RITM) to 23 licensed testing laboratories. ${ }^{4}$ As of September 4, 2020, RITM has helped accredit a total of 117 reverse transcription - polymerase chain reaction (RT-PCR) and cartridge-based PCR laboratories across the country. ${ }^{5}$

Indeed, molecular diagnostic laboratories play a pivotal role in the diagnosis and management of human diseases, including COVID-19. Considering that RT-PCR remains the gold standard for verifying COVID-19 cases, the diagnostic accuracy for this technique is of utmost importance. ${ }^{6}$ Thus, this article aims to present the different sources of contamination in the laboratory setting where RT-PCR assays are conducted, as well as provide efficient, effective and feasible solutions to address these issues, most especially in low- and middle-income countries (LMICs) like the Philippines.

\section{SPECIMEN COLLECTION}

When collecting specimens, it is important to properly identify the sample, collect adequate amount or volume as well as practice standard protocols in the transport and proper storage of biological materials to be tested. ${ }^{6}$

LMICs, however, are confronted with limitations in the availability of PPEs and scarcity of manpower while the number of suspected cases needing confirmatory testing is exponentially growing. With limited resources and excessive workload, compliance with the recommended protocols might be challenging but cannot be discounted since breaching them can be an immediate source of cross-contamination that can jeopardize the accuracy and quality of RT-PCR testing as well as a source of laboratory acquired infections.

For healthcare providers collecting specimens or within 6 feet of patients suspected to be infected with SARS$\mathrm{CoV}-2$, proper infection control must be observed. Prior to specimen collection, all PPEs must be sanitized and worn following the proper sequence. When wearing gloves, it is important to cover part of the forearm while assuring that they remain under the sleeves to minimize skin exposure. Using a second pair of gloves may also be done to cover part of the sleeves. PPEs, including the gown, FFP2 (N95), goggles or face shield, and gloves must be worn all the time. $^{7}$ Male health workers are also advised to shave in order to obtain an adequate mask's adherence to the face. ${ }^{8}$
When collecting the sample, the patient must be seated in a comfortable position with the head resting against a plexiglass divider. After collection, the nasopharyngeal or oropharyngeal swabs are placed in sterile test tubes. The tubes are then properly labelled with the patient's personal data and transported to the laboratory in special containers designated for biohazard materials. ${ }^{8-10}$ Proper labelling, handling, and storage of collected sample is important not only to avoid a false positive result, but a false negative as well. ${ }^{7}$

It is crucial to change gloves and to clean the work area between each collection to prevent cross-contamination. If this is not feasible or is impractical given the limited resources and manpower on top of a demanding workload, an option is to disinfect the gloved hands with $70 \%$ alcohol in a squeeze or spray bottle and then dry with fresh paper towel after each patient. Surfaces of the collection booth, which are made of plastic or metal or sealed with a nonporous cover, should also be disinfected especially when patients made physical contact on the area. WHO has provided guidelines on the use of disinfectants such as sodium hypochlorite or bleach $(0.1 \%$ for general surface disinfection and $1 \%$ for disinfection of sample spills), $62-71 \%$ ethanol, $0.5 \%$ hydrogen peroxide, quaternary ammonium compounds, and phenolic compounds (used according to manufacturer's recommendations). Although less effective, $0.05-0.2 \%$ benzalkonium chloride or $0.02 \%$ chlorhexidine digluconate can also be used. Apart from using the correct disinfectant, contact time, dilution and shelf-life should also be considered. ${ }^{11}$ Alcohol can also be sprayed, but must be wiped only after at least 20 seconds of contact with the surface. Bleach solutions should be prepared fresh each use. ${ }^{11}$ The sample collection boxes or coolers, reusable cold packs, pouches, and racks must also be regularly disinfected. But it must be noted that after disinfection, the technician must wipe the surfaces with paper towel wet with sterile water followed by $70 \%$ alcohol dampened paper towel to prevent residue build-up and PCR inhibition.

When sampling is done, removal of PPEs should follow correct sequence while avoiding contact with external surfaces. The suit, shoes, used gloves, and used mask must be placed in a special waste container. The hands are also cleansed with soap and water or sanitized with alcoholic solution. ${ }^{8}$

\section{ANALYTICAL ISSUES}

Confirmatory laboratory tests through nucleic acid amplification assay is performed for suspected cases. The use of RT-PCR remains the gold standard for testing wherein unique sequences of the SARS-CoV-2 genome are detected. ${ }^{12}$ However, RT-PCR is labor intensive and is an inherently complex assay requiring experience in all aspects of testing, and thus limiting the capacity for quick turnaround time from sample collection to the availability of results. This bottleneck may lead to long wait periods and an exponential demand for testing. ${ }^{6}$ With the increasing number of suspected cases needing confirmatory diagnostic testing, laboratory personnel are forced to work under severe pressure in high-throughput settings with an insurmountable workload and with limited 
access to personal protective equipment (PPE). ${ }^{13}$ Due to the shortage of a trained clinical laboratory workforce, especially in resource-limited countries, diagnostic centers are forced to hire additional laboratory personnel who have limited experience and technical knowledge and skills of molecular assays, especially in processing specimens, interpreting the results, identifying errors, and troubleshooting, in order to meet the demand of increased testing. ${ }^{14}$ Thus, the vulnerability of laboratory medical services to diagnostic errors, including crosscontamination, is increased and with the tendency for generating false-positive results that can compromise the health of the patient and disrupt the efficacy of public health policies and public health response, surveillance programs, and restrictive measures for containing the outbreak. ${ }^{15}$ In worst cases, a false-positive result may entail unnecessary treatment and may undermine available workforce, especially if the patient is working as a public servant and is forced to self-isolate. Meanwhile, a false-negative result can foster rapid human-to-human transmission of the virus due to the failure in the application of restrictive and containment measures as well as in the identification of other suspected cases, especially those exposed to the patient who is infected with SARS-CoV-2. ${ }^{6}$

Thus, the World Health Organization had released guidelines on biosafety in laboratories handling COVID-19 specimens. ${ }^{16}$ Likewise, the Department of Health in the Philippines had released guidelines on how to operate local COVID-19 testing laboratories. ${ }^{17}$ This sets the standard to make sure that tests are reliable, and to promote the safety of those operating the laboratory. Aside from this, some studies suggest that there is a high rate of false negative test results from using the RT-PCR diagnostic kits. ${ }^{18}$ With this, it is important to avoid unnecessary errors particularly in processing samples.

In the laboratory, RT-PCR is a multi-procedural process which makes it susceptible to cross-contamination. After sample collection, RNA is extracted from the specimen to prepare for RT-PCR. The RNA of SARSCoV-2 can be easily transferred from a contaminated gloved hand to the working surface, or to the laboratory environment. ${ }^{19}$ Although WHO guidelines suggest good microbiological practice and procedure, it is not clear on how often laboratory personnel should change gloves. The guidelines are also more focused on protecting the laboratory personnel. To avoid cross-contamination, it is important to promote changing of gloves as frequently as possible, especially if soiled with solutions containing template RNA. Not only in the use of gloves, but the entire set of the PPE should be changed when moving to a different area of the laboratory. Aside from this, materials such as pens, small equipment, tubes, pipette tips, and other consumables should never be brought from RT-PCR to the pre-PCR area. Laboratory guidelines require unidirectional workflow, as such laboratorians and even the cleaning personnel should be reminded to treat each area as a different room to prevent conveying the amplicons to amplification product-free areas. Moreover, the Philippines' DOH guidelines require the separation of pre-PCR room into two areas: (1) specimen handling or sample preparation room and (2) reagent preparation room. The reagent preparation room is a 'template' free environment which also excludes positive internal reaction controls. Provision of different storage areas and freezers for specimens and reagents is highly encouraged.

Another possible source of cross-contamination is the pipetting of patient samples into the PCR plate or strip. Possibly, samples can also be misidentified as positive due to sample misplacement. Thus, proper pipetting and double-checking sample placement while still following aseptic techniques (use of PPE, use of sterile materials, disinfecting work area) when running RT-PCR analysis should always be followed. Cleaning of the work area, pipettors, freezer handles, and other materials using the appropriate decontaminating agent is also a must before and after PCR work. Racks should be immersed in disinfectant for 10 minutes and then dried with clean paper towel. It is also prudent for clinical molecular laboratories to invest on autoclavable pipettors to lessen cross-contaminations.

Increased frequency of disinfection with the use of disinfectants as provided by the WHO guidelines can also be practiced, either every 30 minutes or after processing of COVID-19 samples. ${ }^{11}$ However, for consumables that have been in contact with infectious samples, disposables are recommended.

After RT-PCR analysis, post-PCR is an important step to interpret results for diagnosis. No amplification must be observed in the negative controls provided by the test kit, as well as in the elution buffer (or whichever is appropriate depending on the test kit used) to guarantee that there is no contamination in the process. In case there is possible contamination, the quality of water should be checked, and in some cases contamination of the instrument can also be considered. ${ }^{20}$ To avoid these problems, fresh (unopened) water must be used in each run, and the reagents should be prepared in aliquots in sterile containers once the kit is opened. Proper aseptic technique must be observed until the samples are placed in the machine. When placing samples and controls in the multi-well plate, it is recommended for the controls not to be placed next to each other to make sure that no crosscontamination happens while samples are transferred to their designated wells. It is also highly recommended to assign around 3 or more water controls randomly in the multi-well plate to monitor aseptic pipetting.

It is also worth noting that cross-contamination in the laboratory may not be the only cause of unreliable results. In the early stage of the COVID-19 pandemic, a delay in testing in Europe was caused by a contamination in the test kits. ${ }^{21}$ This problem was also experienced by the Philippines when locally made test kits were found to have contaminated reagents. ${ }^{22}$ Nevertheless, observing proper techniques in COVID-19 testing laboratories should always be strictly followed to avoid unreliable results which are counterproductive in any situation.

Technicians assigned in a COVID-19 testing laboratory can also become infected with the virus and unintentionally contaminate the sample they process and the laboratory environment. Hence, technicians, especially those assigned in the PCR room, are advised to wear goggles or 
face shield and disposable surgical cap and mask, which they must be dispose of in proper bins located in the same room before leaving the area. Disposable lab gowns are highly recommended but may not be practical in low resource areas. Hence, it is advised that laboratory gowns must not be brought home by the technicians but have to be washed and sterilized by their hospital linen and laundry services.

\section{CONCLUSION}

The COVID-19 pandemic has caused a global public health emergency. Although efforts have been made to prevent the spread of the disease, there is still an increasing number of cases each day. Reliable diagnosis through RT-PCR testing plays an important role in the management of the disease. Thus, it is essential to avoid any cross-contamination when handling biological specimens from patients. Proper laboratory practices should always be observed, with the focus on changing gloves as often as possible and changing PPE when moving from one work area to another. Guidelines from the World Health Organization on how to operate laboratories should be strictly followed, as well as those provided by a country's health ministry. Unidirectional workflow in the laboratory while following aseptic technique in each step is crucial in maintaining the reliability of a molecular diagnostic laboratory.

\section{STATEMENT OF AUTHORSHIP}

The authors certified fulfillment of ICMJE authorship criteria.

\section{AUTHOR DISCLOSURE}

The authors declared no conflict of interest.

\section{FUNDING SOURCE}

None.

\section{REFERENCES}

1. Gonzales C. Philippines with highest COVID-19 cases in Southeast Asia? DOH cites different 'settings' (Inquirer). [Online]. Available from: https://newsinfo. inquirer. net/1318069/ph-with-highest-covid-19-casesin-se-asia-doh-cites-different-settings. Accessed 7 August 2020.

2. Mietzner M. Populist anti-scientism, religious polarisation, and institutionalised corruption: how Indonesia's democratic decline shaped its COVID-19 response. J Curr Southeast Asian Affairs. 2020. https:// doi.org/10.1177/1868103420935561.

3. Department of Health. Philippines now has one hundred licensed testing labs. [Online]. Available from: https://www.doh.gov.ph/doh-pressrelease/ PH I L I P P I N ES - N OW-HAS - 100 - L I CE N S E D TESTING-LABS-DOH. Accessed 21 September 2020.

4. World Health Organization. 100 Days of COVID-19 in the Philippines: How WHO supported the Philippine response. [Online]. Available from: https://www.who. int/philippines/news/feature-stories/detail/100-days- of-covid-19-in-the-philippines-how-who-supportedthe-philippine-response. Accessed 21 September 2020.

5. Research Institute for Tropical Medicine. RITM labs surpass quarter-million COVID-19 tests. [Online]. Available from: http://ritm.gov.ph/ritm-labssurpass-quarter-million-covid-19-tests/. Accessed 21 September 2020.

6. Lippi G, Simundic AM, Plebani M. Potential preanalytical and analytical vulnerabilities in the laboratory diagnosis of coronavirus disease 2019 (COVID-19). Clin Chem Lab Med. 2020;58(7):1070-6. PMID: 32172228. https://doi.org/10.1515/cclm-20200285 .

7. Centers for Disease Control and Prevention. Interim guidelines for collecting, handling, and testing clinical specimens for COVID-19. [Online]. Available from: https://www.cdc.gov/coronavirus/2019-ncov/ lab/guidelines-clinical-specimens.html. Accessed 19 September 2020.

8. Piras A, Rizzo D, Longoni E, Turra N, et al. Nasopharyngeal swab collection in the suspicion of Covid-19. Am J Otolaryngol. 2020;41(5):102551. PMID: 32487335. PMCID: PMC7255165. https://doi. org/10.1016/j.amjoto.2020.102551.

9. Irving SA, Vandermause MF, Shay DK, Belongia EA. Comparison of nasal and nasopharyngeal swabs for influenza detection in adults. Clinical Med Res. 2012; 10(4):215-8. PMID: 22723469. PMCID: PMC3494547. https://doi.org/10.3121/cmr.2012.1084.

10. Spencer S, Gaglani M, Naleway A, et al. Consistency of influenza A virus detection test results across respiratory specimen collection methods using realtime reverse transcription-PCR. J Clin Microbiol. 2013; 51(11):3880-2. PMID: 24108606. PMCID: PMC3889789. https://doi.org/10.1128/JCM.01873-13.

11. Loh TP, Horvath AR, Wang CB, et al. Operational considerations and challenges of biochemistry laboratories during the COVID-19 outbreak: an IFCC global survey. Clin Chem Lab Med. 2020;58(9): 1441-9. PMID: 32549122. https://doi.org/10.1515/ cclm-2020-0710.

12. Sahajpal NS, Mondal AK, Njau A, Ananth S, et al. Proposal of RT-PCR-based mass population screening for Severe Acute Respiratory Syndrome Coronavirus 2 (Coronavirus Disease 2020). J Mol Diagnostics. 2020;22(10):1294-9. https://doi.org/10.1016/j.jmoldx. 2020.07.001.

13. Sheridan C. Coronavirus and the race to distribute reliable diagnostics. Nat Biotechnol. 2020;38(4):3824. PMID: 32265548. https://doi.org/10.1038/d41587020-00002-2.

14. Aguilar K. 'Manpower Shortage Hounding Bid to Boost COVID-19 Testing Capacity' (Inquirer). [Online]. Available from: https://newsinfo.inquirer. net/1307576/ manpower-shortage-hounding-bid-toboost-covid-19-testing-capacity. Accessed 6 August 2020.

15. Giri AK, Rana DR. Charting the challenges behind the testing of COVID-19 in developing countries: Nepal as a case study. Biosaf Health. 2020;2(2):536. PMCID: PMC7219426. https://doi.org/10.1016/j. bsheal.2020.05.002.

16. World Health Organization. Laboratory biosafety guidance related to coronavirus disease (COVID-19). 
[Online]. Available from: https://www.who.int/ publications/i/item/ laboratory-biosafety-guidancerelated-to-coronavirus-disease-(covid-19). Accessed 7 August 2020.

17. Department of Health. Guidelines in securing a license to operate a COVID-19 testing laboratory. [Online]. Available from: https://www.doh.gov.ph/node/21040. Accessed 7 August 2020.

18. Younes N, Al-Sadeq DW, AL-Jighefee H, et al. Challenges in laboratory diagnosis of the novel Coronavirus SARS-CoV-2. Viruses. 2020;12(6):582. PMID: 32466458. PMCID: PMC7354519. https://doi. org/10.3390/v12060582.

19. Lv J, Yang J, Xue J, Zhu P, Liu L, Li S. Detection of SARS-CoV-2 RNA residue on object surfaces in nucleic acid testing laboratory using droplet digital PCR. Sci Total Environ. 2020;742:140370. PMID: 32619841. PMCID: PMC7303629. https://doi.org/10.1016/j. scitotenv.2020.140370.
20. van Zyl G, Maritz J, Newman H, Preiser W. Lessons in diagnostic virology: expected and unexpected sources of error. Rev Med Virol. 2019;29(4):e2052. PMID: 31145511. https://doi.org/10.1002/rmv.2052.

21. Mögling R, Meijer A, Berginc N, et al. Delayed Laboratory Response to COVID-19 caused by Molecular Diagnostic Contamination. Emerg Infect Dis. 2020; 26(8):1944-6. PMID: 32433015. PMCID: PMC7392437. https://doi.org/10.3201/ eid2608.201843.

22. Philippine News Agency. UP-NIH test kits recalled due to reagent contamination: FDA. [Online]. Available from: https://www.pna.gov.ph/articles/1 103574. Accessed 8 August 2020.

Disclaimer: This journal is OPEN ACCESS, providing immediate access to its content on the principle that making research freely available to the public supports a greater global exchange of knowledge. As a requirement for submission to the PJP, all authors have accomplished an AUTHOR FORM, which declares that the ICMJE criteria for authorship have been met by each author listed, that the article represents original material, has not been published, accepted for publication in other journals, or concurrently submitted to other journals, and that all funding and conflicts of interest have been declared. Consent forms have been secured for the publication of information about patients or cases; otherwise, authors have declared that all means have been exhausted for securing consent.

\section{Publish in the new PJP. Visit our website: http://philippinejournalofpathology.org}

